\title{
Prime Numbers Calculation Formulas
}

\author{
Ameha Tefera Tessema ${ }^{1}$
}

\begin{abstract}
The application of prime numbers in modern science, especially in computer science, is very wide. Since prime numbers can only divisible by 1 and themselves, they are not factored any further like whole numbers. The problem to calculate all prime numbers using a formula posed for long periods.

Though different formulas to calculate prime numbers were developed by Euler, Fermat, Mersenne and others, the formulas work for limited natural numbers and calculate limited prime numbers. However, in this paper the author wants to show how prime numbers calculated for all values of integers using the following theorem.
\end{abstract}

Theorem 1: Let $x \neq \pm 1$ be integers, then for all $n \neq 1 \in N \quad x^{2 n}+x^{n}-1$ and $(2 x)^{2 n}+\left(\frac{1}{2}\right)(2 x)^{n}-1$ are prime numbers except few odd numbers.

JEL classification numbers: C02, C63, C69

Keywords: Prime numbers, Prime numbers formula, Prime number distribution, Prime number calculation.

${ }^{1}$ Strategic planning, Commercial bank of Ethiopia, Addis Ababa, Ethiopia.

Article Info: Received: April 2, 2021. Revised: April 22, 2021.

Published online: April 29, 2021. 


\section{Introduction}

The nature and properties of Prime numbers were first studied widely by ancient Greek mathematicians. In about 300 BC when Euclid's Elements appeared, several important results about prime numbers had been proved. Later they discovered the fundamental Theorem of Arithmetic, which states that every integer $n>1$ can be represented as a product of primes in only one way, apart from the order of the factors. The last digit of any prime numbers represented by 1,3,7, or 9 . The numbers 2,4,6 and 8 are excluded to ensure that the number is odd and hence is not divisible by the number 2 . Since prime numbers appear in randomly, they tend to avoid having the same last digit for their immediate prime predecessor. Prime numbers are a set of all numbers which are equally divided by 1 and themselves.

Lack of prime number divisor except themselves is still the subject of countless investigation.

Though prime number has great importance in information technology such as public-key cryptography, there is no known useful formula that generate all the prime numbers (Kline,1968). Nowadays, most computer cryptography works by using prime factoring of large numbers which used to encrypt the file that can be publicly known and available because the encryption works so only the prime factors of that large number can be used to decrypt it again. Prime numbers are the most fundamental build blocks of all natural numbers, which are build block of our understanding of the universe. The most widely used applications of prime numbers computing are the RSA encryption system. RSA is a public-key cryptosystem that is widely used for secure data transmission. Prime numbers that are known as infinite in number appeared quite irregularly. They appear within the infinite string of numbers in such a random fashion that devising a functional equation to correctly predict them, infinitely, is believed by many to be an impossible task and the endeavor of calculating prime number using formula found unsuccessful for long periods. Instead of seeking an exact formula for the prime numbers calculation, they considered the counting function $\pi(\mathrm{x})$ and asked for approximations to this function, evidently a new kind of question in number theory (Bateman \& Diamond,1996). If the form of $\mathrm{f}(\mathrm{x})$ function could be obtained exactly we would have the formula to calculate all the prime numbers. Unfortunately until now, it has not been possible to obtain such an exact expression. In 1752 Goldbach showed that no polynomial with an integer coefficient can give a prime number for all integer values (Hardy and Wright,1979 and Mollin,1997). The best known prime numbers formula which accredited to Euler in 1772 is $n^{2}+n+41$ which give prime numbers for all $\mathrm{n}=0$ to 39(Mollin,1997). Another prime number calculation formula, which is $2^{\mathrm{r}}+1$ where $r \equiv 2^{q}$ for $q=0-4$, developed by Fermat and $2^{\mathrm{p}}-1$, where $\mathrm{p}$ is prime numbers, developed by Mersenne (Matiyasevich, 1999 and Rowland, 2008). However, these formulas do not calculate all prime numbers and are valid only for some natural numbers. The objective of the paper is to show how the prime number can be calculated for all integer values using Theorem 1 below. 


\section{Preliminary Notes}

The formulas yield only prime numbers for all integer value $(x)$ where $n \in N$ except for few odd numbers. The formula to calculate prime number by Euler valid only for values starting from 0 up to 39 (Mollin,1997). Fermat and Mersenne formulas to calculate prime number works only for the limited natural numbers and calculate limited prime numbers (Matiyasevich, 1999 and Rowland, 2008). A lot of formulas to calculate prime number developed even though they work only for the first few non-negative values (Apostol, 1976).

However, the prime number calculation formulas developed by this paper, except for few odd numbers, calculate prime numbers for all integer values except \pm 1 and for all natural numbers exponents except 1 . The formulas developed by this paper have a great contribution to mathematics specifically and for science in general. The study of prime numbers and their properties has attracted mathematicians for several centuries because of the use of prime numbers in the different field. (Mabrouk, Hernández-Castro, \& Fukushima, 2011).The formulas do have a new outlook in breaking many security systems based on primes. The importance of prime numbers has increased especially in the field of information technology, i.e., in data security algorithms. The RSA system in cryptography uses prime numbers widely to calculate the public and the private keys. The strength of this system relies upon the difficulty of factoring large numbers - specifically the difficulty associated with the finding of the specific pair of prime numbers selected to create a large integer called the modulus. Diffie-Hellman Key Exchange in cryptography similarly uses prime numbers. It uses a large prime number as a common modulus based on which two entities, say A and B can communicate securely using their private, undisclosed keys (Desai, 2015).

\section{Main results}

Result 1: $x^{2 n}+x^{n}-1$

Result 2: $\frac{1}{2}\left(2 x^{2 n}+x^{n}-2\right)=x^{2 n}+\left(\frac{1}{2}\right) x^{n}-1 \quad$ since the formula work for all even number the value of $x$ should be replaced by $2 x$ and we have the following result

$=(2 x)^{2 n}+\left(\frac{1}{2}\right)(2 x)^{n}-1$

where $n \neq 1, x \neq 1$

\subsection{Advantages}

The formulas calculate prime numbers for all positive and negative integers except 1 or -1 and for all natural numbers except 1 . 


\subsubsection{Cost}

The formulas are handy to use and have no any cost.

Theorem 1: Let $x \neq \pm 1$ be integers, then for all $n \neq 1 \in N \quad x^{2 n}+x^{n}-1$ and $(2 x)^{2 n}+\left(\frac{1}{2}\right)(2 x)^{n}-1$ are prime numbers except few odd numbers.

Proof. Let $n \in N$ (natural numbers) such that $m_{n}, R_{n} \in R$ (real numbers ).

Let

$$
m_{1}=1, m_{2}=\frac{1}{\left(\frac{1}{m_{1} R_{1}}\right)-1}, \quad m_{3}=\frac{1}{\left(\frac{1}{m_{2} R_{2}-m_{1} R_{1}}\right)-\left(\frac{1}{m_{1} R_{1}}\right)+1}
$$

Then it follows that

$$
R_{1}+R_{2}+R_{3}=\left(\frac{1}{m_{1}}\right)\left(m_{1} R_{1}\right)+\left(\frac{1}{m_{2}}\right)\left(m_{2} R_{2}\right)+\left(\frac{1}{m_{3}}\right)\left(m_{3} R_{3}\right)
$$

This can be expressed as

$$
=\left(\frac{1}{m_{1}}\right)\left(0+m_{1} R_{1}\right)+\left(\frac{1}{m_{2}}\right)\left(\left(m_{2} R_{2}-m_{1} R_{1}\right)+m_{1} R_{1}\right)+\left(\frac{1}{m_{3}}\right)\left(\left(m_{3} R_{3}-m_{2} R_{2}+m_{1} R_{1}\right)+\left(m_{2} R_{2}-m_{1} R_{1}\right)\right)
$$

Collecting like terms

$$
\begin{aligned}
& =\left(\frac{1}{m_{1}}+\frac{1}{m_{2}}\right)\left(m_{1} R_{1}\right)+\left(\frac{1}{m_{2}}+\frac{1}{m_{3}}\right)\left(m_{2} R_{2}-m_{1} R_{1}\right)+\left(\frac{1}{m_{3}}\right)\left(m_{3} R_{3}-m_{2} R_{2}+m_{1} R_{1}\right) \\
& =\left(\frac{1}{m_{1} R_{1}}\right)\left(m_{1} R_{1}\right)+\left(\frac{1}{m_{2} R_{2}-m_{1} R_{1}}\right)\left(m_{2} R_{2}-m_{1} R_{1}\right)+\left(\frac{1}{m_{3}}\right)\left(m_{3} R_{3}-m_{2} R_{2}+m_{1} R_{1}\right) \\
& =1+1+\left(\frac{1}{m_{3}}\right)\left(m_{3} R_{3}-m_{2} R_{2}+m_{1} R_{1}\right) \\
& =2+\left(\frac{1}{m_{3}}\right)\left(m_{3} R_{3}-m_{2} R_{2}+m_{1} R_{1}\right)
\end{aligned}
$$

Assume that $R_{1}=x_{1}^{n}, R_{2}=x_{2}^{n}, \frac{1}{m_{2} R_{2}-m_{1} R_{1}}=\frac{-1}{x_{2}^{n}}$ for $m_{3}$, then

$$
x_{1}^{n}+x_{2}^{n}+R_{3}=2+\left(1-\left(\frac{1}{x_{1}^{n}}+\frac{1}{x_{2}^{n}}\right)\right)\left(\frac{R_{3}}{1-\left(\frac{1}{x_{1}^{n}}+\frac{1}{x_{2}^{n}}\right)}-\frac{\left(x_{1} x_{2}\right)^{n}}{1-x_{1}^{n}}+x_{1}^{n}\right)
$$


Then it reduces to

$$
\begin{aligned}
& x_{1}^{n}+x_{2}^{n}=2+\left(1-\left(\frac{1}{x_{1}^{n}}+\frac{1}{x_{2}^{n}}\right)\right)\left(\frac{\left(x_{1} x_{2}\right)^{n}}{x_{1}^{n}-1}+x_{1}^{n}\right) \\
& x_{1}^{n}+x_{2}^{n}=2+\left(\left(x_{1} x_{2}\right)^{n}-\left(x_{1}^{n}+x_{2}^{n}\right)\right)\left(\frac{1}{x_{1}^{n}-1}+\frac{1}{x_{2}^{n}}\right) \\
& \left(x_{1}^{n}+x_{2}^{n}\right)\left(\frac{1}{x_{1}^{n}-1}+\frac{1}{x_{2}^{n}}+1\right)=2+\left(x_{1} x_{2}\right)^{n}\left(\frac{1}{x_{1}^{n}-1}+\frac{1}{x_{2}^{n}}\right) \\
& \left(x_{1}^{n}+x_{2}^{n}\right)\left(\frac{x_{1}^{n}+\left(x_{1} x_{2}\right)^{n}-1}{\left(x_{1}^{n}-1\right)\left(x_{2}^{n}\right)}\right)=\frac{\left(x_{1} x_{2}\right)^{n}\left(x_{1}^{n}+x_{2}^{n}+1\right)-2 x_{2}^{n}}{\left(x_{1}^{n}-1\right)\left(x_{2}^{n}\right)}
\end{aligned}
$$

So that

$$
x_{1}^{n}+x_{2}^{n}=\frac{\left(x_{1} x_{2}\right)^{n}\left(x_{1}^{n}+x_{2}^{n}+1\right)-2 x_{2}^{n}}{x_{1}^{n}+\left(x_{1} x_{2}\right)^{n}-1} \text { for } x_{1}<x_{2}
$$

Let $x_{1}=x_{2}=x$, then

$$
\begin{aligned}
& 2 x^{n}=\frac{\left(x^{2 n}\right)\left(2 x^{n}+1\right)-2 x^{n}}{x^{2 n}+x^{n}-1} \\
& \frac{2 x^{2 n}+x^{n}-2}{x^{2 n}+x^{n}-1}=2 \quad \text { where } \mathrm{x} \neq \pm 1
\end{aligned}
$$

As we can see above $2 x^{2 n}+x^{n}-2$ is twice $x^{2 n}+x^{n}-1$.

Therefore, we have the following formulas

$$
\frac{1}{2}\left(2 x^{2 n}+x^{n}-2\right)=x^{2 n}+\left(\frac{1}{2}\right) x^{n}-1 \text { and } x^{2 n}+x^{n}-1 \text {. }
$$

\section{Conclusion}

The paper introduced a new formulas to calculate prime numbers for all integer values and for all natural numbers exponents. This broaches a new outlook in number theory specifically and mathematics in general.

Because primes are of the highest importance to number theorists and mathematic in general, they are the building blocks of whole numbers whose product can be written by one or more prime number, and important to the world because their odd mathematical properties make them perfect for our current uses.

Large prime numbers are used obviously in cryptosystems. This study will help researchers to uncover the critical formulas of prime numbers that may researchers were not able to explore. Thus, the new theory on prime numbers formulas to 
calculate prime numbers may be arrived at. Since the formulas work for all integer values, mathematician should further investigate to which exponent natural number as the formulas perfectly work to calculate prime numbers. The limitation of the formulas is that they calculate rarely odd number.

\section{References}

[1] Morris Kline (1968). Mathematics in modern world, W.h. freeman and co., Sanfrancisco.

[2] Paul T. Bateman and Harold G. Diamond (1996). A Hundred Years of Prime Numbers, The American Mathematical Monthly, 103:9, 729-741, DOI: $10.1080 / 00029890.1996 .12004813$

[3] Hardy, G. H. and Wright, E. M. (1979). An Introduction to the Theory of Numbers, 5th ed. Oxford science publication, England: Clarendon Press, pp. 18 and 22.

[4] R.A.Mollin (1997). Prime-producing Quadratics, The America Mathematical Monthely, Vol.104, No.6, PP.529-544. https://doi.org/10.2307/2975080

[5] Matiyasevich, Yuri V. (1999). Formulas for Prime Numbers, in Tabachnikov, Serge (ed.), Kvant Selecta: Algebra and Analysis, II, American Mathematical Society, pp. 13-24.

[6] Rowland, Eric S. (2008). A Natural Prime-Generating Recurrence, Journal of Integer Sequences 11: 08.2.8, arXiv:0710.3217, Bibcode:2008JIntS..11...28R.

[7] Apostol, Thomas M. (1976). Introduction to Analytic Number Theory, New York, Springer, pp.74-105.

[8] E. Mabrouk, Hernández-Castro, J.C. and Fukushima, M. (2011). Prime number generation using memetic programming. Artif Life Robotics 16, 53-56. https://doi.org/10.1007/s10015-011-0890-3

[9] Tejash Desai (2015). Application of Prime Numbers in Computer Science and the Algorithms Used To Test the Primality of a Number, international Journal of Science and Research, Vol. 4, Issue 9. 